\title{
Sympathetic Neural Mechanisms in Obstructive Sleep Apnea
}

Virend K. Somers, Mark E. Dyken, Mary P. Clary, and Francois M. Abboud

Departments of Internal Medicine and Neurology and the Cardiovascular Center, University of Iowa College of Medicine, Iowa City, Iowa 52242

\begin{abstract}
Blood pressure, heart rate, sympathetic nerve activity, and polysomnography were recorded during wakefulness and sleep in 10 patients with obstructive sleep apnea. Measurements were also obtained after treatment with continuous positive airway pressure (CPAP) in four patients. Awake sympathetic activity was also measured in 10 age- and sexmatched control subjects and in 5 obese subjects without a history of sleep apnea.

Patients with sleep apnea had high levels of nerve activity even when awake $(P<0.001)$. Blood pressure and sympathetic nerve activity did not fall during any stage of sleep. Mean blood pressure was $92 \pm 4.5 \mathrm{mmHg}$ when awake and reached peak levels of $116 \pm 5$ and $127 \pm 7 \mathrm{mmHg}$ during stage II sleep $(n=10)$ and rapid eye movement (REM) sleep $(n=5)$, respectively $(P<0.001)$. Sympathetic activity increased during sleep $(P=0.01)$ especially during stage II (133 $\pm 9 \%$ above wakefulness; $P=0.006)$ and $R E M$ $(141 \pm 13 \% ; P=0.007)$. Peak sympathetic activity (measured over the last $10 \mathrm{~s}$ of each apneic event) increased to $299 \pm 96 \%$ during stage II sleep and to $246 \pm 36 \%$ during REM sleep (both $P<0.001$ ). CPAP decreased sympathetic activity and blood pressure during sleep $(P<\mathbf{0 . 0 3})$.

We conclude that patients with obstructive sleep apnea have high sympathetic activity when awake, with further increases in blood pressure and sympathetic activity during sleep. These increases are attenuated by treatment with CPAP. (J. Clin. Invest. 1995. 96:1897-1904.) Key words: sympathetic nerve activity $\bullet$ blood pressure $\cdot$ hypertension - hypoxia
\end{abstract}

\section{Introduction}

Patients with obstructive sleep apnea are at increased risk for hypertension, myocardial ischemia, and stroke (1-5), and have an increased cardiovascular morbidity and mortality $(6,7)$. They also have a high incidence of electrocardiographic changes suggestive of ischemia during sleep; these changes are largely

Address correspondence to Virend K. Somers, M.D., Ph.D., Cardiovascular Division, Department of Internal Medicine, University of Iowa College of Medicine, 200 Hawkins Drive, Iowa City, IA 52242. Phone: 319-353-8570; FAX: 319-353-6343.

Received for publication 23 September 1994 and accepted in revised form 28 June 1995.

J. Clin. Invest.

(C) The American Society for Clinical Investigation, Inc. 0021-9738/95/10/1897/08 \$2.00

Volume 96, October 1995, 1897-1904 corrected by therapy with continuous positive airway pressure $(\text { CPAP })^{1}$ (8).

Autonomic and hemodynamic responses to obstructive sleep apnea (9) are complex and include the effects of apnea, hypoxia, hypercapnia, the Mueller maneuver (inspiration against a closed glottis), and arousal. Hypoxia and hypercapnia act synergistically to increase sympathetic activity $(10,11)$; this increase is especially marked during apnea when the sympathoinhibitory influence of the pulmonary afferents is eliminated $(12,13)$.

Sleep, by contrast, is associated with a marked decline in both blood pressure and sympathetic activity during non-rapid eye movement (REM) sleep (14-16). Thus, chemoreflex-mediated sympathetic excitation and blood pressure increases during apneic episodes (17) might be opposed by sympathoinhibitory and blood pressure-lowering mechanisms that govern nonREM sleep.

To investigate the net effect of these opposing autonomic and hemodynamic influences, we measured blood pressure, heart rate, and sympathetic nerve activity during wakefulness and sleep in patients with obstructive sleep apnea. We also examined the effects of treatment with CPAP.

\section{Methods}

We studied 18 patients ( 14 males, 4 females) referred for evaluation of sleep apnea. Informed written consent was obtained from each subject. These studies were approved by the institutional Human Use Committee. Technically excellent recordings of sympathetic activity during wakefulness and sleep were obtained in 10 subjects ( 7 males, 3 females; age $\sim 45 \pm 12$ yr). Patient characteristics are described in Table I. Studies were abandoned in six subjects because of inability to obtain nerve recordings $(n=3)$ or instability of nerve recordings during the early stages of sleep $(n=3)$. Two subjects did not have obstructive sleep apnea. Recordings during wakefulness were also obtained in 10 ageand sex-matched control subjects. These subjects denied symptoms of obstructive sleep apnea, were free of significant medical illness, and were on no medications, except for one subject with glaucoma (on Timolol eye drops). Volunteers of the same sex and of similar age to patients with obstructive sleep apnea underwent microneurographic recordings of sympathetic nerve activity. If a successful nerve recording was obtained, these subjects then underwent polysomnography to rule out significant sleep apnea (i.e., apnea-hypopnea index $>10$ ). We also obtained measurements of sympathetic activity and blood pressure during sleep in three of these control subjects. In addition, we obtained measurements of sympathetic activity during wakefulness in five obese subjects, who were free of symptoms suggestive of obstructive sleep apnea.

Experimental methods and data analysis were similar to those used in an earlier study on sleep in normal subjects (16). Studies started at about 10 p.m. and finished at about 4 a.m. Sympathetic nerve activity

1. Abbreviations used in this paper: CPAP, continuous positive airway pressure; OSA, obstructive sleep apnea; REM, rapid eye movement; RESP, respiration; SNA, sympathetic nerve activity. 
Table I. Patient and Control Characteristics (Sex-matched Control Data Shown in Parentheses)

\begin{tabular}{|c|c|c|c|c|c|c|c|c|}
\hline Patient & Sex & Age & $\begin{array}{l}\text { Body mass } \\
\text { index }\end{array}$ & Hypertension & Medications & $\begin{array}{l}\text { Apnea-hypopnea } \\
\text { index }\end{array}$ & $\begin{array}{c}\text { Burst } \\
\text { frequency }\end{array}$ & Burst frequency \\
\hline & & $y r$ & $\mathrm{~kg} / \mathrm{m}^{2}$ & & & & bursts/min & bursts/100 heart beats \\
\hline 1 & $\mathrm{~F}$ & $57(56)$ & $36.0(23.6)$ & $+(-)$ & Propranolol (-) & 68 -severe $(0)$ & $38(32)$ & $62(52)$ \\
\hline 2 & $\mathbf{F}$ & $61(60)$ & $37.9(25.0)$ & $+(-)$ & Verapamil (-), Thyroxine & 21-moderate $(0)$ & $55(32)$ & $79(56)$ \\
\hline 3 & $\mathrm{~F}$ & $49(44)$ & $44.0(27.5)$ & $+(-)$ & Diltiazem (-), Thyroxine & 20-moderate (3) & $78(47)$ & $97(51)$ \\
\hline $4^{*}$ & $\mathbf{M}$ & $48(48)$ & $33.3(20.2)$ & $-(-)$ & Allopurinol (-) & 22-moderate $(0)$ & $67(31)$ & $81(44)$ \\
\hline $5 *$ & $\mathbf{M}$ & $46(47)$ & $52.8(23.2)$ & $-(-)$ & Phenytoin (-) & 84-severe (1) & $68(51)$ & $84(75)$ \\
\hline $6^{*}$ & $\mathbf{M}$ & $27(27)$ & $46.3(28.8)$ & $-(-)$ & - & 30 -moderate $(0)$ & $52(25)$ & $62(38)$ \\
\hline $7 *$ & $\mathbf{M}$ & $55(59)$ & $49.9(27.4)$ & $+(-)$ & $\begin{array}{l}\text { INH Pyridoxine (Timolol } \\
\text { eye drops) }\end{array}$ & 55 -severe $(0)$ & $72(39)$ & $96(59)$ \\
\hline 8 & $\mathbf{M}$ & $49(52)$ & $32.4(26.9)$ & $-(-)$ & $-(-)$ & 10-mild (4) & $53(42)$ & $71(66)$ \\
\hline 9 & $\mathbf{M}$ & $32(34)$ & $47.0(23.9)$ & $-(-)$ & $-(-)$ & 27-moderate (1) & $41(18)$ & $51(29)$ \\
\hline 10 & $\mathbf{M}$ & $30(33)$ & $36.7(26.7)$ & $-(-)$ & $-(-)$ & 107 -severe $(0)$ & $62(24)$ & $80(44)$ \\
\hline
\end{tabular}

* Data also obtained after CPAP. INH, isoniazid.

was measured using direct multiunit intraneural recordings of efferent sympathetic discharge to muscle blood vessels (microneurography) (18, 19). A tungsten microelectrode (shaft diameter $200 \mu \mathrm{M}$ and tip of 1$5 \mu \mathrm{M}$ ) was inserted into the sympathetic nerve fascicles in the peroneal nerve posterior to the fibular head. A reference electrode was inserted subcutaneously $\sim 3 \mathrm{~cm}$ away. Electrical signals obtained from the sympathetic nerve fascicles were amplified (50,000-90,000 times), filtered (bandpass filter, band width of 700-2,000 Hz), and passed through a resistance-capacitance integrating network with a time constant of 0.1 $\mathrm{s}$, providing a mean voltage display of sympathetic nerve activity. Sympathetic bursts were identified by inspection of the mean voltage neurogram, and sympathetic activity was calculated as bursts per minute, and bursts per 100 heart beats, and by measurements of total burst amplitude per minute, expressed as arbitrary units (19). Measurements of bursts per 100 heart beats allows a comparison of baseline sympathetic nerve activity levels between subjects $(20-25)$.

Measurements of sympathetic nerve activity were normalized so that the mean value for wakefulness was $100 \%$. Measurements of nerve activity for the different sleep stages were expressed as a percentage of the values for wakefulness (16). Measurements of nerve activity during sleep were made throughout a given sleep stage (overall nerve activity) as well as during the last $10 \mathrm{~s}$ of each apneic event during a given sleep stage (peak nerve activity).

Heart rate was measured using an electrocardiogram. Continuous measurements of beat-by-beat blood pressure were obtained by intraarterial measurement $(n=3)$ or using the Finapres system (26). Measurements obtained using this system have been shown to correspond closely to intraarterial blood pressure measurements both at rest and during rapid changes in blood pressure (27). Subjects were fitted with a Finapres cuff appropriate to finger size. The recording arm position was fixed with the aid of sand bags and an arm board. The Finapres was switched off for $\sim 5-10$ min every $2 \mathrm{~h}$ for subject comfort.

Complete polysomnographic recordings were obtained including oxygen saturation, nasal and oral air flow (measured by temperaturesensitive thermocouples), chest movement, electromyogram, electroencephalogram, and electrocardiogram. All variables in this study were recorded continuously. Severity of sleep apnea was defined on the basis of the apnea-hypopnea index. Apnea refers to cessation of both nasal and oral airflow, and hypopnea refers to a reduction in airflow to $<50 \%$ of baseline in association with oxygen desaturation. To be considered significant, abnormal respiratory events had to persist for a maximum of $10 \mathrm{~s}$ or had to occur in association with an arousal and/or a decrease in oxygen saturation by $3 \%$ or more. Obstruction was confirmed by persistent respiratory effort recorded by thoracic-abdominal strain gauge and the electromyogram. The apnea-hypopnea index indicates the number of respiratory irregularities per sleep hour and is calculated as follows (28): (total number of apneas + hypopneas)/(total sleep time in minutes) $\times 60 / 1$. An index of $<5$ is normal, 5-20 suggests mild sleep apnea, $20-50$ is moderate, and $>50$ indicates severe sleep apnea.

Sleep stages were scored based on the recommendations of Rechtstaffen and Kales (29). Normal sleep consists of REM and nonREM sleep. Non-REM sleep is further subdivided into stages I, II, III, and IV, indicating progressively deeper levels of sleep and characterized by progressively slower frequency and increased voltage activity on the electroencephalogram. REM sleep is characterized by the onset of low voltage mixed frequency activity on the electroencephalogram, associated with loss of muscle tone and intermittent discrete episodes of rapid eye movements $(30-32)$.

Because of frequent and often continuously repetitive apneic episodes, all subjects exhibited brief arousals from sleep on termination of apnea. The arousals prevented progression to deeper sleep stages in five subjects, who remained in stage II sleep for prolonged periods. Four subjects progressed to stages III, IV, and REM sleep. Because of frequent momentary arousals secondary to apneic episodes, it was not possible to clearly separate stages III and IV sleep and these stages were analyzed together and are referred to as stage IV sleep. In one subject, REM sleep, with persistence of obstructive apneas, was achieved after administration of low grade CPAP at $6 \mathrm{mmHg}$ and lasted only $5 \mathrm{~min}$. Thus, measurements for stage I and II sleep were obtained in all 10 subjects, for stage IV sleep in 4 subjects, and for REM sleep in 5 subjects. Duration of sleep during which complete data were obtained averaged $2.4 \pm 0.4 \mathrm{~h}$ per subject. In four subjects with moderate to severe obstructive sleep apnea, measurements were also taken during therapeutic levels of CPAP administered the same night. Duration of recordings during CPAP administration averaged $2.1 \pm 0.5 \mathrm{~h}$ per subject.

Data were analyzed from 4-min periods of wakefulness, stage II, stage IV, and REM sleep (16). Because of the shorter duration of stage I sleep, 2-min periods of this stage were analyzed. Three randomly selected 4-min epochs (2-min epochs for stage I) were analyzed for each of these sleep stages. Thus, for each subject, measurement for each of wakefulness, stage II, stage IV, and REM was calculated from a total of $12 \mathrm{~min}$ of measurement for each stage in each subject ( $6 \mathrm{~min}$ for stage I). The exception was in the one subject in whom REM sleep lasted only $5 \mathrm{~min}$. Thus, only one 4-min epoch could be obtained during REM sleep for this subject.

Sympathetic nerve recordings remained remarkably stable through the night despite the arousals, and segments of data analyzed were interrupted by minor adjustment of the recording electrode on only $1.6 \pm 0.4$ occasions per subject (including CPAP recordings). This stabil- 


\section{AWAKE}

NORMAL


$10 \sec$

Figure 1. Recordings of sympathetic nerve activity (SNA) during wakefulness in patients with obstructive sleep apnea (OSA) and matched controls showing high levels of SNA in patients with sleep apnea.

ity was achieved by careful stabilization of the leg from which recordings were obtained, based on our experience in earlier studies (16). In addition, because many patients were obese, a specially designed elongated $(50 \mathrm{~mm})$ electrode was used. This was inserted into the nerve at an acute angle to the skin, thus enabling most of the electrode to be embedded in subcutaneous tissue, providing very stable recordings despite frequent arousals. Studies were discontinued in patients in whom nerve site recordings were unstable during wakefulness or early stages of sleep.

We noted high levels of sympathetic burst frequency in patients with sleep apnea. We therefore further examined whether obesity could be the explanation for this. We obtained measurements of sympathetic nerve activity in five obese subjects, four males and one female, $31 \pm 7$ yr old, with body mass index of $40.3 \pm 3.2 \mathrm{~kg} / \mathrm{m}^{2}$, similar to the body mass index of the patients with sleep apnea $\left(41.6 \pm 2.3 \mathrm{~kg} / \mathrm{m}^{2}\right)$. All obese subjects denied a history suggestive of obstructive sleep apnea and were on no medications. One obese subject had mild hypertension.

Statistical analysis was conducted using a two-way ANOVA. The two factors in the analysis were subject and sleep stage with more than one observation per stage per subject. Comparisons of interest were tested by defining mean contrasts. These were then tested using the test statistic with standard errors for the test computed from variance estimates derived from the ANOVA (33). Comparison of wakefulness measurements between sleep apneic and normal control subjects and between sleep apneic and obese control subjects was conducted using an unpaired $t$ test. The $P$ values reported were based on a two-tailed test. Significance was assumed at the $5 \%$ level. Results are expressed as mean \pm standard error.

\section{Results}

Sympathetic burst frequency when awake was higher in patients with obstructive sleep apnea $(59 \pm 14$ bursts/min or $76 \pm 5$ bursts / 100 heart beats) than in normal controls ( $34 \pm 3$ bursts/ min or $50 \pm 4$ bursts $/ 100$ heart beats; $P<0.001$ ) (Fig. 1). Sympathetic burst frequency during wakefulness was also higher in patients with obstructive sleep apnea than in obese controls, in whom burst frequency was $24 \pm 5$ bursts/min or
$34 \pm 8$ bursts $/ 100$ heart beats (both $P<0.001$ ). Repetitive episodes of apnea occurred continuously throughout all of sleep in most patients with obstructive sleep apnea, resulting in large oscillations in blood pressure and sympathetic activity synchronous with the apneas (Figs. 2 and 3). As apnea progressed, blood pressure rose gradually, in association with increasing sympathetic activity. On termination of apnea, sympathetic activity ceased abruptly, and blood pressure increased, reaching levels as high as $240 / 130 \mathrm{mmHg}$ in some subjects. Termination of apnea was also accompanied by transient increases in muscle tone and evidence of brief arousal on the electroencephalogram (Fig. 3). Only one patient had no apneas for substantial periods of sleep (during stage IV). These oscillations and arousals were absent during this time.

Because of the blood pressure oscillations, we measured average blood pressure during sleep as well as peak (highest blood pressure) for each minute analyzed. Because of continuous occurrence of apneas throughout sleep, almost all nerve activity occurred during the periods of apnea with suppression of sympathetic activity on release of apnea, when blood pressure reached peak levels. Despite periods of sympathetic activity suppression and despite the already high levels of sympathetic activity during wakefulness, sympathetic activity measured for all of sleep increased to $125 \pm 9 \%$ of levels measured during wakefulness $(P=0.01)$.

Analysis of individual sleep stages showed that the sympathetic activity increases occurred mainly during stage II $(133 \pm 9 \% ; P=0.006)$ and REM sleep $(141 \pm 13 \% ; P=0.007)$, when apnea severity and oxygen desaturation were greatest (Fig. 4). Oxygen saturation was $96 \pm 3 \%$ when awake and fell significantly during all sleep stages. Peak sympathetic activity, at the end of each apneic event, reached $198 \pm 55 \%$ during stage I, $299 \pm 96 \%$ in stage II, $212 \pm 24 \%$ in stage IV, and $246 \pm 36 \%$ in REM (all $P<0.002$ ) (Fig. 4). It is unlikely that changes in recording electrode position contributed to our findings since electrode repositioning was required on less than two occasions per subject, and burst frequency (adjusted for heart rate ] 18[) remained stable from wakefulness through the different sleep stages. Burst frequency was $59 \pm 3$ bursts/min when awake and $53 \pm 3,52 \pm 3,52 \pm 4$, and $55 \pm 4$ bursts/min during stages I, II, IV, and REM, respectively (all not significant except for stage II where $P=0.04$ ). Heart rate fell from $77 \pm 2.4$ beats $/ \mathrm{min}$ when awake to $73 \pm 2.4,72 \pm 2.4,71 \pm 2.8$, and $72 \pm 2.7$ beats/ min during stages I, II, IV, and REM, respectively (all $P$ $<0.04$ ). Burst frequency per 100 heart beats was $76 \pm 3.4$ when awake and $72 \pm 3.5,73 \pm 3.4,73 \pm 4.7$, and $76 \pm 4.3$ during stages I, II, IV, and REM, respectively (all not significant).

The peak levels of sympathetic activity occurred toward the end of apnea and immediately before surges in blood pressure. Blood pressure did not fall during sleep. Mean blood pressure averaged $92 \pm 4.5$ during wakefulness, $95 \pm 4.5$ in stage I, $96 \pm 4.5$ in stage II, and $89 \pm 5.2$ in stage IV (Fig. 4). Stage IV data include measurements from the one patient relatively free of apneas during stage IV sleep, in whom blood pressure during stage IV sleep was $14 \mathrm{mmHg}$ lower than when awake. Mean blood pressure during REM sleep increased to $104 \pm 5 \mathrm{mmHg}$ $(P=0.003)$ (Fig. 4). Peak mean blood pressure was higher than wakefulness for all sleep stages, reaching levels of $116 \pm 5$ $\mathrm{mmHg}$ during stage II and $127 \pm 7 \mathrm{mmHg}$ during REM (both $P<0.0001$ ).

No significant correlations were detected between sympa- 
SNA



RESP
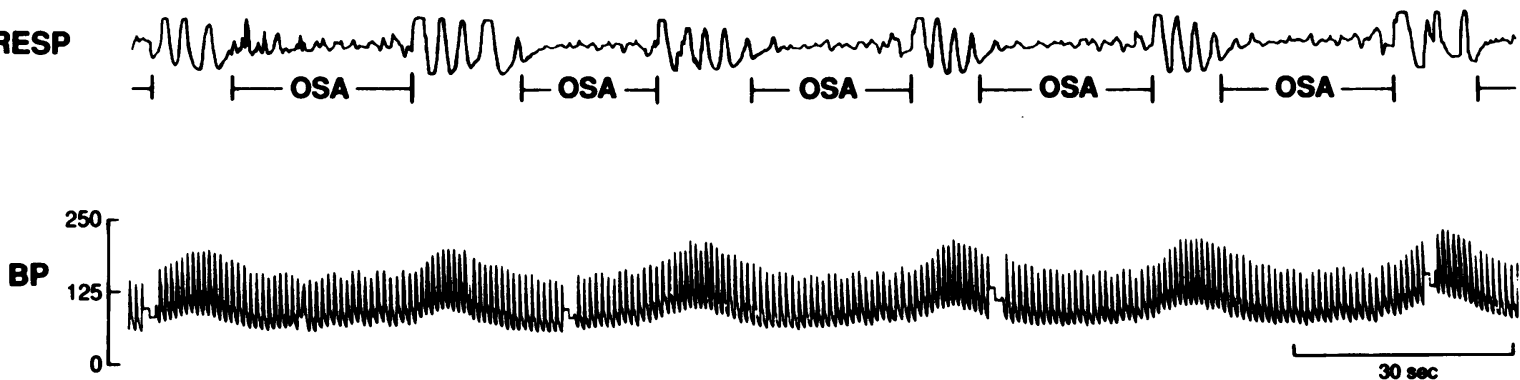

Figure 2. Recordings of SNA, respiration (RESP), and blood pressure $(B P)$ during $3 \mathrm{~min}$ of stage II sleep, showing incessant oscillations in blood pressure and sympathetic activity in response to the repetitive OSAs. These oscillations occurred continuously during sleep, throughout all sleep stages.

thetic nerve activity (both during wakefulness and sleep) and body mass index, apnea-hypopnea index, or blood pressure.

Blood pressure, heart rate, and sympathetic activity were also recorded during sleep in three of the normal control subjects. Changes in these variables during sleep were qualitatively consistent with changes reported in earlier studies (14-16). Compared with wakefulness, during non-REM slow-wave sleep, mean blood pressure fell from $90 \pm 6$ to $83 \pm 7 \mathrm{mmHg}$, heart rate fell from $62 \pm 4$ to $59 \pm 2$ beats/min, sympathetic burst frequency fell from $32 \pm 2$ to $20 \pm 7$ bursts/min, and sympathetic burst amplitude decreased to $52 \pm 20 \%$ of values recorded during wakefulness.

In four patients with moderate to severe sleep apnea, subjects were awakened after several hours of sleep and treatment with CPAP was instituted the same night. Airway pressure levels were gradually increased during sleep to minimize episodes of obstructive apnea. Oxygen saturation during sleep (with correction for sleep stage) was $20 \pm 5.5 \%$ higher with CPAP ( $P$ $=0.04$ ) and remained above $90 \%$ for all sleep stages. In these four patients, mean blood pressure during sleep, without CPAP,

EOG

EEG

EMG

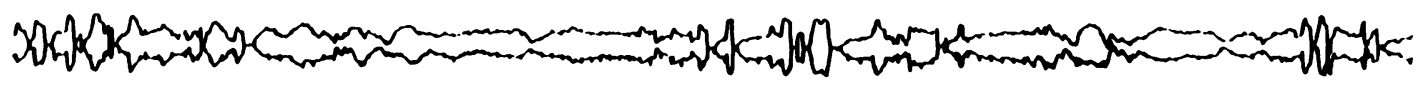

EKG



SNA



RESP
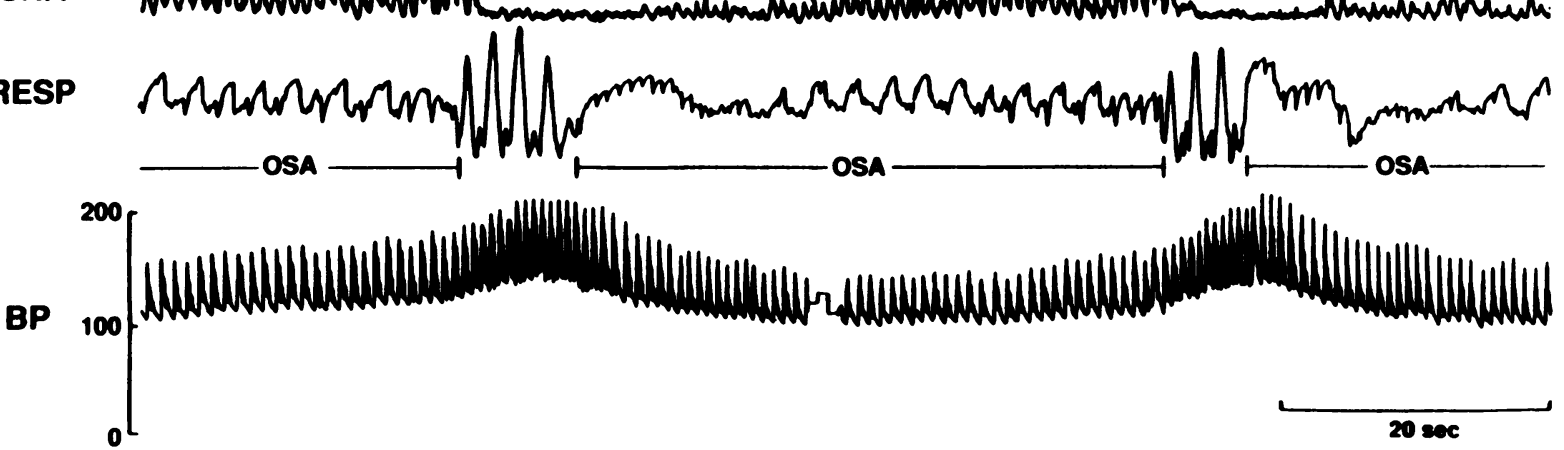

Figure 3. Superimposed recordings of the electrooculogram ( $E O G)$, electroencephalogram (EEG), electromyogram (EMG), electrocardiogram $(E K G)$, SNA, RESP, and BP during REM sleep in a patient with OSA. BP during REM, even during the lowest phases $(\sim 160 / 105 \mathrm{mmHg})$, was higher than in the awake state $(130 / 75 \mathrm{mmHg})$. BP surges at the end of the apneic periods reached levels as high as $220 / 130 \mathrm{mmHg}$. EOG shows the sharp eye movements characteristic of REM sleep. Increase in muscle tone $(E M G)$ and cessation of rapid eye movements toward the end of the apneic period indicates arousal from REM sleep (arrows). 


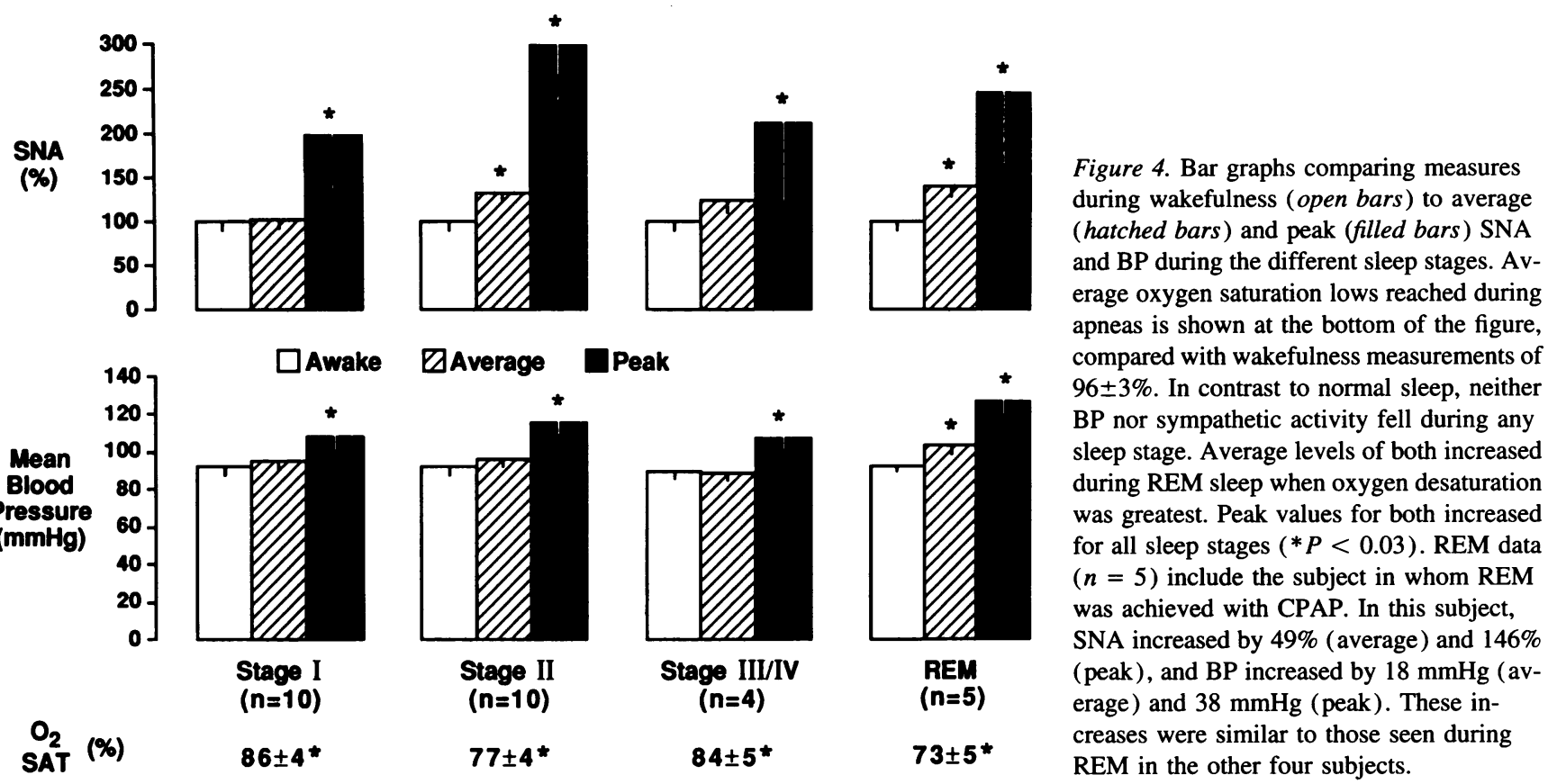

increased over wakefulness by $11 \pm 3 \mathrm{mmHg}(P=0.008)$, and peak blood pressure increased by $36 \pm 4 \mathrm{mmHg}(P<0.001)$. Average and peak sympathetic activity during sleep were $120 \pm 11$ and $190 \pm 15 \%$ of wakefulness values, respectively. Blood pressure and sympathetic activity measured at the start of the study, before sleep, did not differ significantly from measurements when awake before starting CPAP. After treatment with CPAP, average blood pressure increased during sleep by only $4 \pm 3 \mathrm{mmHg}$ and peak blood pressure increased by only $10 \pm 4 \mathrm{mmHg}$ (both not significantly different from wakefulness). Average and peak sympathetic activities decreased during sleep and were $30 \pm 10(P=0.02)$ and $4 \pm 14 \%$ less than wakefulness values, respectively (Figs. 5-7).

\section{Discussion}

These studies indicate that sympathetic nerve discharge is very high in sleep apneic patients even when awake. The sympathetic nerve and blood pressure profile during sleep is dominated by responses to episodes of obstructive sleep apnea that occur continuously throughout sleep. The organized pattern of sleep stage-related changes in blood pressure and sympathetic activity evident during sleep in normal humans (14-16) is disrupted. Despite sleep stage and the higher blood pressure during sleep, there is an increase in sympathetic activity during sleep in patients with sleep apnea, even though these patients already have very high levels of nerve activity when awake. Apneic episodes result in progressive increases in sympathetic nerve activity, these increases being most marked toward the end of the apnea. With cessation of apnea and resumption of breathing, there is an abrupt termination of sympathetic activity and increases in blood pressure. In contrast to sleep in normal humans, when blood pressure and sympathetic nerve activity decline significantly during non-REM sleep (14-16, 34), both sympathetic activity and blood pressure reach very high levels during sleep in patients with sleep apnea. Attenuation of obstructive apneas by treatment with positive airway pressure limits oxygen desatu- ration and attenuates both sympathetic activity and blood pressure increases during sleep.

Several reports have described transient increases in blood pressure during sleep apneic episodes $(35,36)$. There are few studies of neural mechanisms in sleep apnea. In a qualitative report on daytime studies in sleep apneic patients, Hedner et al. (17) reported high and fluctuating levels of sympathetic activity, associated with blood pressure oscillations. Sympathetic activity during wakefulness was higher than that in age-matched controls. Sleep stages were not reported.

Potential limitations of our study include, first, drug therapy in some of our subjects. However, high levels of activity during wakefulness and sleep were also evident in the seven patients who were not on vasoactive medications. Furthermore, chronic antihypertensive treatment with beta blockade does not increase nerve activity (37). The effect of chronic antihypertensive medication on blood pressure responses to apnea is not known.

Second, although microneurographic recordings of sympathetic nerve activity were obtained from a single limb, there is a remarkable and pronounced synchrony in simultaneous measures of nerve activity from different sympathetic fascicles regardless of which limb or muscle bed the fascicles innervate (20). This is true at rest and during apnea $(20,21)$. Thus microneurographic measurements reliably indicate the central drive to post-ganglionic sympathetic neurons to the vascular bed of skeletal muscle, which constitutes $\sim 45 \%$ of body mass and thus plays a key role in regulating arterial pressure (38). Microneurographic measurements of burst frequency correlate with plasma norepinephrine levels between individuals (39) and have the crucial advantage of providing continuous moment-bymoment measures of sympathetic activity.

The reason for the very high levels of sympathetic discharge, even in the awake state, in sleep apneic patients is not clear. Factors such as age, obesity, mild oxygen desaturation, carbon dioxide retention, and hypertension may be implicated. Age and hypertension alone may not fully explain the high levels of activity (17). In a study investigating age-related changes in 


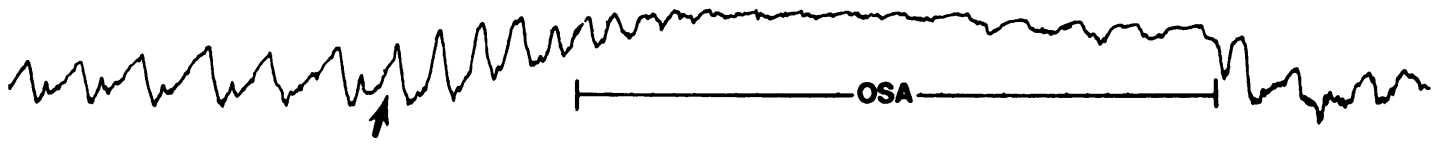

BP

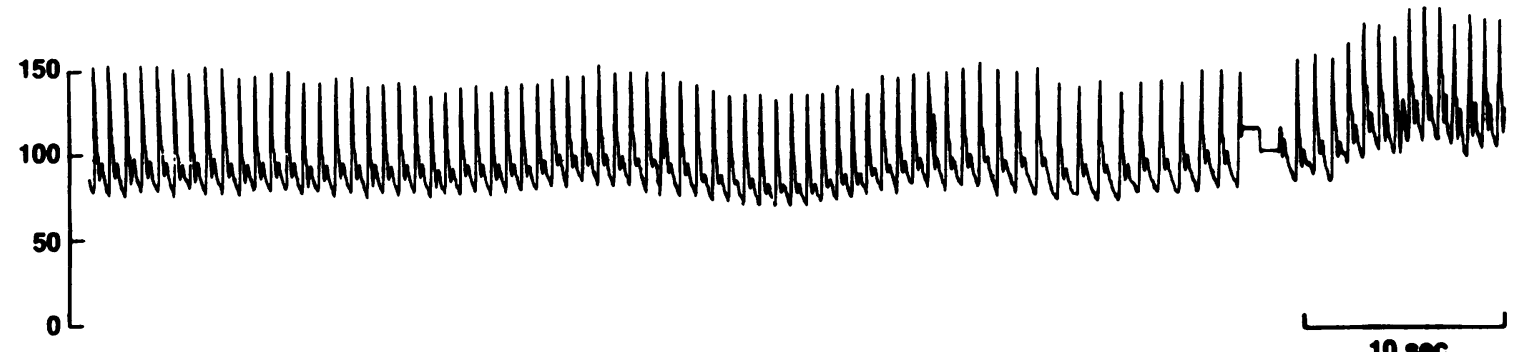

Figure 5. Recordings of the electrocardiogram (EKG), SNA, RESP, and BP in a sleep apneic patient undergoing CPAP therapy during REM sleep. The arrow indicates reduction of CPAP from 8 to $6 \mathrm{mmHg}$, allowing the development of obstructive apnea, with consequent increased SNA and BP. In normal humans, BP and SNA are highest during REM sleep. In patients with OSA, apneic events during REM result in further increases in both SNA and BP.

sympathetic nerve activity in hypertensive patients, Yamada et al. (22) noted that nerve activity was highest $(63 \pm 5$ bursts/ 100 heart beats $)$ in older $(59 \pm 1.0 \mathrm{yr})$ hypertensive pa- tients. This level of nerve activity is still substantially lower than $76 \pm 5$ bursts $/ 100$ heart beats noted in our younger sleep apneic patient group. Obesity may partly explain the increased
AWAKE

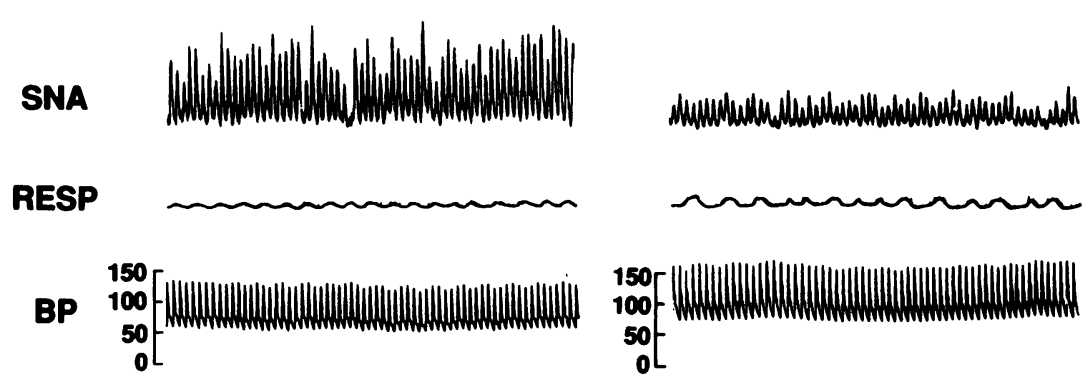

SNA

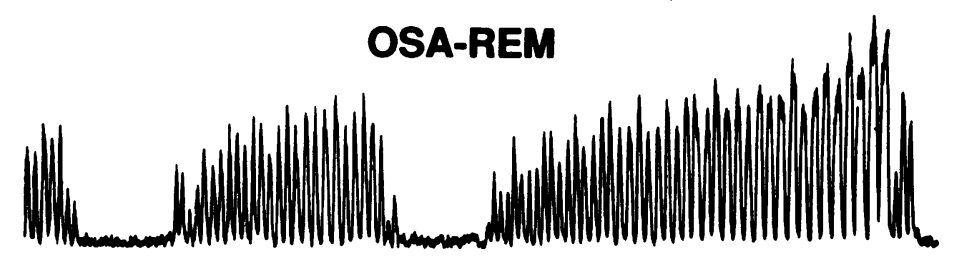

RESP

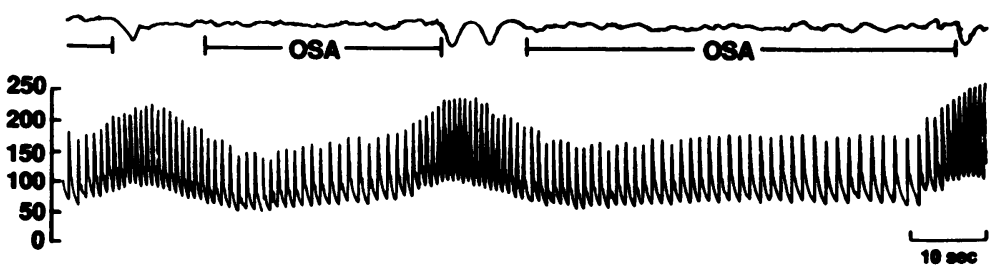

Figure 6. Recordings of SNA, RESP, and intraarterial BP in the same subject when awake, with obstructive sleep apnea during REM sleep and with elimination of obstructive apnea by CPAP therapy during REM sleep. SNA is very high during wakefulness, but increases even further secondary to obstructive apnea during REM. BP increases from $130 / 65 \mathrm{mmHg}$ when awake to $256 / 110$ $\mathrm{mmHg}$ at the end of apnea. Elimination of apneas by CPAP results in decreased nerve activity and prevents BP surges during REM sleep. 



Figure 7. Bar graphs comparing changes in average and peak BP and sympathetic activity in four patients during sleep (with correction for sleep stage) as compared with measurements when awake. Open bars indicate changes during OSA and hatched bars indicate the changes during treatment with CPAP $\left({ }^{*} P<0.04\right)$.

sympathetic activity in patients with sleep apnea. However, body mass index has been shown to correlate inversely with plasma norepinephrine levels in humans and animals (40). In obese subjects without a history of sleep apnea, sympathetic burst frequency when awake is significantly lower than levels seen in patients with sleep apnea. Thus, obesity alone is unlikely to explain the increased sympathetic activity noted in patients with sleep apnea. Even if obesity does contribute significantly to high levels of sympathetic activity when awake, obstructive sleep apnea results in even higher levels of sympathetic activity (and blood pressure) during sleep. These increases are partially corrected by CPAP therapy, indicating that it is obstructive sleep apnea, rather than obesity per se, that induces sympathetic activation and blood pressure increases during sleep. Carbon dioxide tension was measured in six of our sleep apneic subjects (by blood gas or end tidal methods) and ranged between 39 and $44 \mathrm{mmHg}$. Oxygen saturation in our subjects averaged $96 \pm 3 \%$. Oxygen desaturation to levels of $91 \%$ do not elicit increases in sympathetic activity in humans (10). We have shown previously that hypoventilation-induced hypercapnia (increases of $8 \mathrm{mmHg}$ in $\mathrm{pCO} 2$ ) and accompanying oxygen desaturation to $97 \%$ have little effect on sympathetic nerve activity (41). Thus, hypoxia and hypercapnia are not likely explanations for high levels of sympathetic activity during wakefulness in our subjects.

The oscillations in sympathetic discharge and blood pressure during sleep are explained by chemoreflex mechanisms and their interactions with baroreflexes and the pulmonary afferents $(10,11,42)$. Oxygen desaturation and carbon dioxide retention during sleep apnea both act to increase sympathetic discharge by stimulation of the peripheral and central chemoreflexes (11). With progression of apnea, both of these stimuli increase in intensity, resulting in increased sympathetic discharge to muscle (and probably splanchnic and renal) vascular beds. This vasoconstriction also helps to maintain blood pressure during the apneic episode when cardiac output is decreased because of decreased venous return due to cessation of breathing and the Mueller maneuver $(43,44)$. With termination of apnea and resumption of breathing, there is a tachycardia and an increase in venous return to the heart and, thus, increased cardiac output (43). This increased cardiac output is superimposed upon peripheral vasoconstriction resulting in surges in blood pressure to levels of $240 / 130 \mathrm{mmHg}$ in some subjects. The abrupt termination of sympathetic activity during resumption of breathing is probably due to a combination of factors. First, breathing and stimulation of the pulmonary afferents inhibit sympathetic nerve activity (10-12). Second, the blood pressure increase acting via the baroreflexes further suppresses sympathetic discharge (42). Third, resumption of breathing diminishes hypoxic and hypercapnic chemoreflex stimulation. Fourth, we have shown previously that during REM sleep in normal humans brief increases in muscle tone are associated with abrupt sympathetic inhibition (16). Similar, but more marked increases in muscle tone at the end of apneic events may, at least during REM, contribute to sympathetic inhibition in patients with sleep apnea (Fig. 3). Thus, reflex and hemodynamic interactions involving the chemoreflexes, the pulmonary afferents, the Mueller maneuver (45), venous return, cardiac output, muscle tone, and the baroreflexes all interact to result in repetitive and potentially harmful fluctuations in blood pressure and sympathetic discharge. Arousal from sleep for a brief period at the end of apnea may also be implicated in the responses we describe (46).

The increased sympathetic nerve activity during sleep occurs mainly during apnea episodes and is most intense toward the end of the apnea when hypoxia, hypercapnia, and respiratory acidosis are most profound and immediately before rapid changes in cardiac afterload. These findings suggest an explanation for recurrent episodes of pulmonary edema in patients with obstructive sleep apnea (47). Chronic exposure to these neurohumoral stresses, occurring repetitively each night, may result in adverse cardiac and vascular alterations over many years.

Treatment of obstructive apnea by CPAP attenuates blood pressure and sympathetic increases during sleep. Although CPAP blunts the blood pressure increase during sleep, blood pressure does not fall to below wakefulness levels, as is seen in sleep in normal subjects (14-16). This may be due to CPAP attenuating, but not eliminating, the obstructive apneas. Nevertheless, the lower blood pressure and sympathetic activity and higher oxygen saturation after CPAP indicate a likely mechanism to explain the beneficial effects of CPAP in preventing electrocardiogram changes suggestive of ischemia in patients with sleep apnea $(8)$ and improving cardiac function in heart failure patients with obstructive sleep apnea (48).

\section{Acknowledgments}

We thank Linda Bang for typing the manuscript, James Kinney for technical assistance, and Dr. Bridget Zimmerman for statistical advice. 
These studies were supported by grants HL-07121 and HL-14388 from the National Heart, Lung, and Blood Institute of the National Institutes of Health, and by the Council for Tobacco Research.

\section{References}

1. Lavie, P., R. Ben-Yosef, and A. E. Rubin. 1984. Prevalence of sleep apnea syndrome among patients with essential hypertension. Am. Heart J. 108:373376.

2. Kales, A., R. J. Cadieux, L. C. Shaw III, A. Vela-Bueno, E. O. Dixler D. W. Schneck, T. W. Locke, and C. R. Soldatos. 1984. Sleep apnea in hypertensive population. Lancet. 2:1005-1008.

3. Koskenvuo, M., J. Kaprio, M. Partinen, H. Langinvanio, S. Sarna, and K. Heikkila. 1985. Snoring as a risk factor for hypertension and angina pectoris. Lancet. 1:89-95.

4. Palomaki, H., M. Partinin, S. Juvela, and M. Kaste. 1989. Snoring as a risk factor for sleep-related brain infarction. Stroke. 10:1311-1315.

5. Dyken, M. E., V. K. Somers, T. Yamada, H. P. Adams, Jr., and M. D. Zimmerman. 1992. Investigating the relationship between sleep apnea and stroke. Sleep Research. 2:30a. (Abstr.)

6. Bliwise, D. L., N. G. Bliwise, M. Partinen, A. M. Pursley, and W. C. Dement. 1988. Sleep apnea and mortality in an aged cohort. Am. J. Public Health. 78:544-547.

7. He, J., M. H. Kryger, F. J. Zorick, W. Conway, and T. Roth. 1988. Mortality and apnea index in obstructive sleep apnea. Experience in 385 male patients Chest. 94:9-14.

8. Hanly, P., Z. Sasson, N. Zuberi, and K. Lunn. 1993. ST segment depression during sleep in obstructive sleep apnea. Am. J. Cardiol. 71:1341-1345.

9. Shepard, J. W., Jr. 1990. Cardiopulmonary consequence of obstructive sleep apnea. Mayo Clin. Proc. 65:1250-1259.

10. Somers, V. K., D. C. Zavala, A. L. Mark, and F. M. Abboud. 1989 Influence of ventilation and hypocapnia on sympathetic nerve responses to hypoxia in normal humans. J. Appl. Physiol. 67:2095-2100.

11. Somers, V. K., D. C. Zavala, A. L. Mark, and F. M. Abboud. 1989 Contrasting effects of hypoxia and hypercapnia on ventilation and sympathetic activity in humans. J. Appl. Physiol. 67:2101-2106.

12. Somers, V. K., A. L. Mark, and F. M. Abboud. 1988. Potentiation of sympathetic nerve responses to hypoxia in borderline hypertensive subjects. $\mathrm{Hy}$ pertension (Dallas). 11:608-612.

13. Somers, V. K., A. L. Mark, and F. M. Abboud. 1988. Sympathetic activation by hypoxia and hypercapnia-implications for sleep apnea. Clin. Exp. Hypertens. Part A Theory Pract. A10(Suppl. 1):413-422.

14. Hornyak, M., M. Cejnar, M. Elam, M. Matousek, and B. G. Wallin. 1991 Sympathetic muscle nerve activity during sleep in man. Brain. 114:1281-1295.

15. Okada, H., S. Iwase, T. Mano, Y. Sugiyama, and T. Watanabe. 1991. Changes in muscle sympathetic nerve activity during sleep in humans. Neurology. 41:1961-1966.

16. Somers, V. K., M. E. Dyken, A. L. Mark, and F. M. Abboud. 1993. Sympathetic nerve activity during sleep in normal humans. N. Engl. J. Med. 328:303-307.

17. Hedner, J., H. Ejnell, J. Sellgren, T. Hedner, and G. Wallin. 1988. Is high and fluctuating muscle nerve sympathetic activity in the sleep apnea syndrome of pathogenetic importance for the development of hypertension? J. Hypertens. 6:S529-S531.

18. Wallin, G. 1983. Intraneural recording and autonomic function in man. In Autonomic Failure. R. Bannister, editor. Oxford University Press, London. 3651 .

19. Mark, A. L., R. G. Victor, C. Nerhed, and B. G. Wallin. 1985. Microneurographic studies of the mechanisms of sympathetic nerve responses to static exercise in humans. Circ. Res. 57:462-469.

20. Sundlof, G., and B. G. Wallin. 1977. The variability of muscle nerve sympathetic activity in resting recumbent man. J. Physiol. 272:383-397.

21. Wallin, B. G., D. Burke, and S. C. Gandevia. 1992. Coherence between the sympathetic drives to relaxed and contracting muscles of different limbs of human subjects. J. Physiol. 455:219-233.

22. Yamada, Y., E. Miyajima, O. Tochikubo, T. Matsukawa, and M. Ishii. 1989. Age-related changes in muscle sympathetic nerve activity in essential hypertension. Hypertension (Dallas). 13:870-877.

23. Miyajima, E., Y. Yamada, Y. Yoshida, T. Matsukawa, H. Shionoiri, O. Tochikubo, and M. Ishii. 1991. Muscle sympathetic nerve activity in renovascular hypertension and primary aldosteronism. Hypertension (Dallas). 17:1057- 1062.

24. Svedenhag, J., B. G. Wallin, G. Sundlof, and J. Henriksson. 1984. Skeletal muscle sympathetic activity at rest in trained and untrained subjects. Acta Physiol. Scand. 120:499-504.

25. Scherrer, U., S. F. Vissing, J. J. Morgan, J. A. Rollins, R. S. A. Tindall, R. A. Hanson, P. K. Mohanty, and R. G. Victor. 1990. Cyclosporine-induced sympathetic activation and hypertension after heart transplantation. N. Engl. J. Med. 323:693-699.

26. Wesseling, K. H., J. J. Settels, and B. El Wit. 1986. The measurement of continuous finger arterial pressure noninvasively in stationary subjects. In Biological and Physiological Factors in Cardiovascular Disease. T. H. Schmidt, T. M. Dembroski, and G. Blumchen, editors. Springer-Verlag, Berlin/Heidelberg. 355375.

27. Parati, G., R. Casadei, A. Groppelli, M. DiRienzo, and G. Mancia. 1989. Comparison of finger and intra-arterial blood pressure monitoring at rest and during laboratory testing. Hypertension (Dallas). 13:647-655.

28. Bornstein, S. K. 1982. Respiratory monitoring during sleep: polysomnography. In Sleeping and Waking Disorders: Indications and Technique. C. Guilleminault, editor. Butterworth-Heinemann Ltd., London. 183-212.

29. Rechtstaffen, A., and A. Kales. 1968. A manual of standardized terminology, techniques and scoring system for sleep stages of human subjects. Public Health Service, US Government Printing. 1-12.

30. Kelly, D. D. 1991. Sleep and dreaming. In Principles of Neuroscience. E. R. Kandel, J. H. Schwartz, and T. M. Jessell, editors. Elsevier/North-Holland, New York. 648-658.

31. Dement, W., and N. Leitman. 1957. Cyclic variations in EEG during sleep and their relation to eye movements, body motility and dreaming. Electroencephalogr. Clin. Neurophysiol. 9:673-690.

32. Mancia, G., and A. Zanchetti. 1980. Cardiovascular regulation during sleep. In Physiology in Sleep. J. Orem, editor. Academic Press, New York. 2 50.

33. Snedecor, G. W., and W. G. Cochran. 1980. Statistical Methods. 7th ed. lowa State University Press, Ames, IA. 265-267.

34. Coccagna, G., M. Mantovani, F. Brignani, A. Manzini, and E. Lugaresi 1971. Arterial pressure changes during spontaneous sleep in man. Electroencephalogr. Clin. Neurophysiol. 31:277-281.

35. Tilkian, A. G., C. Gulleminault, J. S. Schroeder, K. L. Lehrman, F. B. Simmons, and W. C. Dement. 1976. Hemodynamics in sleep-induced apnea Studies during wakefulness and sleep. Ann. Intern. Med. 85:714-719.

36. Motta, J., C. Gulleminault, J. S. Schroeder, and W. C. Dement. 1978 Tracheostomy and hemodynamic changes in sleep-induced apnea. Ann. Intern. Med. 89:454-458.

37. Wallin, B. G., G. Sundlof, E. Stromgren, and H. Aberg. 1984. Sympathetic outflow to muscle during treatment of hypertension with metoprolol. Hypertension (Dallas). 6:557-562.

38. Shepherd, R. F. J., and J. T. Shepherd. 1992. Control of blood pressure and the circulation in man. In Autonomic Failure. R. Bannister and C. J. Mathias, editors. Oxford University Press, Oxford. 78-93.

39. Wallin, B. G., G. Sundlof, B. M. Eriksson, P. Dominiak, H. Grobecker, and L. E. Lindblad. 1981. Plasma noradrenaline correlates to sympathetic muscle nerve activity in normotensive man. Acta Physiol. Scand. 111:69-73.

40. Peterson, H. R., M. D. Rothschild, C. R. Weinberg, R. D. Fell, K. R. McLeisch, and M. A. Pfeifer. 1988. Body fat and the activity of the autonomic nervous system. N. Engl. J. Med. 318:1077-1083.

41. Anderson, D. E., V. K. Somers, M. P. Clary, C. A. Sinkey, and E. A. Anderson. 1991. Muscle sympathetic nerve and hemodynamic responses during hypoventilation induced hypercapnia in humans. Soc. Neurosci. Abstr. 86:201.

42. Somers, V. K., A. L. Mark, and F. M. Abboud. 1991. Interactions of baroreceptor and chemoreceptor reflex control of sympathetic nerve activity in normal humans. J. Clin. Invest. 87:1953-1957.

43. Guilleminault, C., J. Motta, F. Mihm, and K. Melvin. 1986. Obstructive sleep apnea and cardiac index. Chest. 89:331-334.

44. Katzenberg, C., M. Olajos, E. Morkin, and S. Goldman. 1986. Effects of changes in airway pressure on the left ventricle and left atrium of dogs. Cardiovasc. Res. 20:853-862.

45. Somers, V. K., M. E. Dyken, and J. L. Skinner. 1993. Autonomic and hemodynamic responses and interactions during the Mueller maneuver in humans. J. Auton. Nerv. Syst. 44:253-259.

46. Pinto, J. M. B., E. Garpestad, J. W. Weiss, D. M. Bergan, and D. A. Kirby. 1993. Hemodynamic changes associated with obstructive sleep apnea followed by arousal in a porcine model. J. Appl. Physiol. 75:1439-1443.

47. Chaudhary, B. A., D. S. Ferguson, and W. A. Speir. 1982. Pulmonary edema as a presenting feature of sleep apnea syndrome. Chest. 82:122-124.

48. Malone, S., P. P. Liu, R. Holloway, R. Rutherford, A. Xie, and T. D. Bradley. 1991. Obstructive sleep apnoea in patients with dilated cardiomyopathy: effects of continuous positive airway pressure. Lancet. 338:1480-1484. 